\title{
Inverse Radon transform and the transverse-momentum dependent functions
}

\author{
I. V. Anikin $\oplus^{1, *}$ and L. Szymanowski $\oplus^{2, \dagger}$ \\ ${ }^{1}$ Bogoliubov Laboratory of Theoretical Physics, JINR, 141980 Dubna, Russia \\ ${ }^{2}$ National Centre for Nuclear Research (NCBJ), 02-093 Warsaw, Poland
}

(Received 18 September 2019; published 26 November 2019)

\begin{abstract}
We revisit the standard representation of the (inverse) Radon transform, which is well known in the mathematical literature. We extend this representation to the case involving the parton distributions. We have found the new additional contribution, which is essentially related to the generalized transversemomentum dependent parton distribution and double-distribution functions. We discuss the possible relationship of this term with the Sivers function.
\end{abstract}

DOI: $10.1103 /$ PhysRevD.100.094034

\section{INTRODUCTION}

The investigation of the quark-gluon dynamics based on both perturbative and nonperturbative methods is one of the most important subjects of hadron phenomenology. Recently, due to the new kind of accelerators, different exclusive hard reactions have begun to be available for study. The special interests are related to the study of generalized parton distributions (GPDs), double distributions (DD) and (generalized) distribution amplitudes [(GDAs) DAs], which are most useful to extract new information on the composite hadron structure [1-7].

In [8], the relations between the GPDs and DDs in the context of both the direct and inverse Radon transforms have been studied for the first time. This finding becomes extremely useful for the further investigations of the Radon transform applications; see, e.g., [9] and [10-13] for the recent studies on the different aspects of the inverse Radon transforms in terms of GPDs.

The Radon transformations have a long history of application in different fields: tomography, astronomy, etc. Special attempts have been directed to the inverse problem, that is, how to reconstruct the function from its projections. One of the main problems is associated with the theorem that states that any function with restricted and compact support can be uniquely determined or reconstructed by the infinite set of projections only (see, e.g., $[14,15])$. This is at odds with the practical use where we deal with only the finite set of projections. For the mathematical community, the mentioned

\footnotetext{
*anikin@theor.jinr.ru

†Lech.Szymanowski@ncbj.gov.pl
}

Published by the American Physical Society under the terms of the Creative Commons Attribution 4.0 International license. Further distribution of this work must maintain attribution to the author(s) and the published article's title, journal citation, and DOI. Funded by SCOAP ${ }^{3}$. ill posedness of the inverse Radon transform is closely related to this fact. In contrast, in the present paper, we focus on the physical aspects of the inverse Radon transformation problem in the context of GPDs (we refer the readers to [9] for the modern recent studies).

The content of the present paper is as follows. We revisit the (inverse) Radon transforms that connect the DDs with GPDs. We have found a new term that, as we show, is essentially related to the $T$-odd $k_{\perp}$-dependent parton distributions. We demonstrate that the restrictions applied on the support of the DDs may lead to the corresponding restrictions for the support of GPDs.

The paper is logically divided into two parts: the first part is devoted to the rather mathematical abstract issues related to the (inverse) Radon transforms. We also discuss the problem of the new term existence. The second part is concentrated on the direct physical application of the new contribution to the process descriptions through the transverse-momentum dependent parton distributions.

\section{GETTING STARTED: THE FOURIER AND RADON TRANSFORMS}

For pedagogical reasons, we shortly recall some important definitions and relations used for the Radon transforms. All details regarding the different aspects of the Radon transforms can be found in [14]. Also, in this section, we consider the general aspects of the (inverse) Radon transforms for the unbounded, restricted, and localized support of the function $f$, which is a base of the Fourier and Radon transformations.

\section{A. The case of unbounded support of homogeneous function $f$}

Let us begin with the Fourier transform that helps us to introduce the Radon transform. For an arbitrary 
two-dimensional homogeneous function $f(\overrightarrow{\mathbf{x}})$, the direct Fourier transform takes the form of

$$
\mathcal{F}[f](\overrightarrow{\mathbf{q}})=\int_{-\infty}^{+\infty} d^{2} \overrightarrow{\mathbf{x}} e^{-i(\overrightarrow{\mathbf{q}}, \overrightarrow{\mathbf{x}}\rangle} f(\overrightarrow{\mathbf{x}}) .
$$

Notice that, in general, both $\mathcal{F}[f](\overrightarrow{\mathbf{q}})$ and $f(\overrightarrow{\mathbf{x}})$ can be the complex functions, i.e., $\{\mathcal{F}[f](\overrightarrow{\mathbf{q}}), f(\overrightarrow{\mathbf{x}})\} \in \mathbb{C}$.

As the first step, we assume the support of function $f(\overrightarrow{\mathbf{x}})$ to be unbounded. It is convenient to choose the polar coordinates for the vector $\overrightarrow{\mathbf{q}}\left(\overrightarrow{\mathbf{q}}=\lambda \overrightarrow{\mathbf{n}}_{\varphi}\right.$ with the normal vector $\overrightarrow{\mathbf{n}}_{\varphi}=(\cos \varphi, \sin \varphi), \varphi \in[0 ; 2 \pi]$, and $\left.\lambda=|\overrightarrow{\mathbf{q}}| \in[0 ;+\infty]\right)$. Inserting the integral unit (the normalization constants have been absorbed into the corresponding integration measures),

$$
\int_{-\infty}^{+\infty}(d t) \delta(t-\langle\overrightarrow{\mathbf{q}}, \overrightarrow{\mathbf{x}}\rangle)=1
$$

we can rewrite Eq. (1) as

$$
\begin{aligned}
\mathcal{F}[f]\left(\lambda \overrightarrow{\mathbf{n}}_{\varphi}\right)= & \int_{-\infty}^{+\infty} d^{2} \overrightarrow{\mathbf{x}} e^{-i \lambda\left\langle\overrightarrow{\mathbf{n}}_{\varphi}, \overrightarrow{\mathbf{x}}\right\rangle} f(\overrightarrow{\mathbf{x}}) \\
& \times \int_{-\infty}^{+\infty}(d t) \delta\left(t-\lambda\left\langle\overrightarrow{\mathbf{n}}_{\varphi}, \overrightarrow{\mathbf{x}}\right\rangle\right) .
\end{aligned}
$$

Making use of the replacement, $\tau=t / \lambda$, we get

$\mathcal{F}[f]\left(\lambda \overrightarrow{\mathbf{n}}_{\varphi}\right) \equiv \mathcal{F}[f](\lambda, \varphi)=\int_{-\infty}^{+\infty}(d \tau) e^{-i \lambda \tau} \mathcal{R}[f](\tau, \varphi)$,

where

$$
\mathcal{R}[f](\tau, \varphi)=\int_{-\infty}^{+\infty} d^{2} \overrightarrow{\mathbf{x}} f(\overrightarrow{\mathbf{x}}) \delta\left(\tau-\left\langle\overrightarrow{\left.\left.\mathbf{n}_{\varphi}, \overrightarrow{\mathbf{x}}\right\rangle\right)}\right.\right.
$$

defines the direct Radon transformation of $f(\overrightarrow{\mathbf{x}})$. If we now perform the rotation of the coordinate system as

$$
\left\{\begin{array}{l}
p=x_{1} \cos \varphi+x_{2} \sin \varphi, \\
s=-x_{1} \sin \varphi+x_{2} \cos \varphi,
\end{array}\right.
$$

Eq. (5) takes the following form,

$$
\begin{aligned}
\mathcal{R}[f](\tau, \varphi)= & \int d p d s \delta(\tau-p) \\
& \times f(p \cos \varphi-s \sin \varphi, p \sin \varphi+s \cos \varphi) \\
= & \int_{L(\tau, \varphi)} d s f\left(x_{1}(\tau, \varphi ; s), x_{2}(\tau, \varphi ; s)\right),
\end{aligned}
$$

where $L(\tau, \varphi)$ denotes the line given by $\tau-\left\langle\overrightarrow{\mathbf{n}}_{\varphi}, \overrightarrow{\mathbf{x}}\right\rangle=0$; the new coordinate $s$ is pointed along the line while the new coordinate $p$ is perpendicular to the line. Hence, one can see that the Radon transform can also be defined through the line integration as

$$
\mathcal{R}[f](\tau, \varphi)=\int_{L(\tau, \varphi)} d s(\overrightarrow{\mathbf{x}}) f(\overrightarrow{\mathbf{x}})
$$

Equation (4) refers to the Fourier slice theorem [14] and it means that the one-dimensional Fourier image of the Radon transform of $f$ with respect to the radial (offset) parameter gives the two-dimensional Fourier transform of $f$, while the angular (slope) parameter $\varphi$ remains untouched. In other words, the angular parameter $\varphi$ plays the role of both the polar coordinate in $q$-plane and the Radon slope parameter.

For our further purposes, it also useful to introduce an alternative notation for the Radon transform as

$$
\mathcal{R}[f](\tau, \varphi) \equiv \mathcal{R}_{\tau, \varphi}[f(\overrightarrow{\mathbf{x}})],
$$

which has been used in Sec. IV.

From Eq. (5), we can see that the Radon transform possesses the symmetry property in the form of

$$
\mathcal{R}[f]\left((-)^{k} \tau, \varphi\right)=\mathcal{R}[f](\tau, \varphi+k \pi), \quad k \in \mathbb{Z} .
$$

Taking into account the symmetry property (10), one can also write down that

$$
\begin{aligned}
& \mathcal{F}[f](\lambda, \varphi) \\
& =\int_{0}^{+\infty}(d \tau)\left\{e^{-i \lambda \tau} \mathcal{R}[f](\tau, \varphi)+e^{i \lambda \tau} \mathcal{R}[f](\tau, \varphi+\pi)\right\} .
\end{aligned}
$$

We stress that the Radon transform $\mathcal{R}[f](\tau, \varphi+\pi)$ can be different from the Radon transform $\mathcal{R}[f](\tau, \varphi)$ depending on the basic properties of function $f$.

As the angular parameter $\varphi$ is the same for both the Fourier and Radon transforms, a single full rotation in $q$-plane, $0 \leq \varphi \leq 2 \pi$, to cover some (unbounded or bounded) domain generates the two rotations in $x$-plane for the Radon transform.

To derive the inverse Radon transform, at first we invert the Fourier transform (1). We have

$$
\begin{aligned}
f(\overrightarrow{\mathbf{x}}) & =\int_{-\infty}^{+\infty} d^{2} \overrightarrow{\mathbf{q}} e^{+i\langle\overrightarrow{\mathbf{q}}, \overrightarrow{\mathbf{x}}\rangle} \mathcal{F}[f](\overrightarrow{\mathbf{q}}) \\
& =\int_{0}^{+\infty} d \lambda \lambda \int_{0}^{2 \pi} d \varphi e^{+i \lambda\left\langle\overrightarrow{\mathbf{n}}_{\varphi}, \overrightarrow{\mathbf{x}}\right\rangle} \mathcal{F}[f](\lambda, \varphi),
\end{aligned}
$$

where the polar coordinates have been used again. Using the relation (4) and performing the replacement, $\tau-\left\langle\overrightarrow{\mathbf{n}}_{\varphi}, \overrightarrow{\mathbf{x}}\right\rangle=\eta$, Eq. (12) can be presented as

$$
\begin{aligned}
f(\overrightarrow{\mathbf{x}})= & \int_{0}^{+\infty} d \lambda \lambda \int_{0}^{2 \pi} d \varphi \\
& \times \int_{-\infty}^{+\infty}(d \eta) e^{-i \lambda \eta} \mathcal{R}[f]\left(\eta+\left\langle\overrightarrow{\mathbf{n}}_{\varphi}, \overrightarrow{\mathbf{x}}\right\rangle, \varphi\right),
\end{aligned}
$$

given that it remains to implement the integration over the variable $\lambda$ in Eq. (13). To do this, we observe that the 
integration variable $\lambda$ can be traded for the derivative over $\eta$ that acts on the exponential function

$$
\lambda e^{-i \lambda \eta}=i \frac{\partial}{\partial \eta} e^{-i \lambda \eta}
$$

Moreover, the integration over $\lambda$, which is singled out, should be regularized by $\eta \rightarrow \eta-i \varepsilon$,

$$
\begin{aligned}
i \frac{\partial}{\partial \eta} \int_{0}^{+\infty} d \lambda e^{-i \lambda(\eta-i \varepsilon)} & \equiv 2 \pi \frac{\partial}{\partial \eta} \delta_{-}(\eta) \\
& =-\frac{\mathcal{P}}{\eta^{2}}+i \pi \frac{\partial}{\partial \eta} \delta(\eta) .
\end{aligned}
$$

Thus, we finally derive for the inverse Fourier transform expressed through the Radon transform

$$
\begin{aligned}
f(\overrightarrow{\mathbf{x}})= & f_{A}(\overrightarrow{\mathbf{x}})+f_{S}(\overrightarrow{\mathbf{x}}) \\
= & -i \pi \int_{-\infty}^{+\infty}(d \eta) \int_{0}^{2 \pi} d \varphi\left[\frac{\partial}{\partial \eta} \mathcal{R}[f]\left(\eta+\left\langle\overrightarrow{\mathbf{n}_{\varphi}}, \overrightarrow{\mathbf{x}}\right\rangle, \varphi\right)\right] \delta(\eta) \\
& -\int_{-\infty}^{+\infty}(d \eta) \frac{\mathcal{P}}{\eta^{2}} \int_{0}^{2 \pi} d \varphi \mathcal{R}[f]\left(\eta+\left\langle\overrightarrow{\mathbf{n}}_{\varphi}, \overrightarrow{\mathbf{x}}\right\rangle, \varphi\right) .
\end{aligned}
$$

Notice that the second term in Eq. (16) is a standard one, while the first term in Eq. (16) looks, in the most general case, artificial due to the symmetry property of the Radon transformation provided that (a) the $\eta$-integration region is (symmetric-) unbounded and (b) the angular variable $\varphi$ has been varied in the full region between 0 and $2 \pi$.

Indeed, using Eq. (5), we can rewrite Eq. (16) in the equivalent form as (if $f$ is a regular function)

$$
\begin{aligned}
f(\overrightarrow{\mathbf{x}}) & =f_{A}(\overrightarrow{\mathbf{x}})+f_{S}(\overrightarrow{\mathbf{x}}) \\
& =-i \pi \int_{0}^{2 \pi} d \varphi \int_{-\infty}^{+\infty}\left(d^{2} \overrightarrow{\mathbf{y}}\right)\left[\left\langle\overrightarrow{\mathbf{n}_{\varphi}}, \vec{\nabla}\right\rangle f(\overrightarrow{\mathbf{y}})\right] \delta\left(\left\langle\overrightarrow{\mathbf{n}}_{\varphi},(\overrightarrow{\mathbf{x}}-\overrightarrow{\mathbf{y}})\right\rangle\right) \\
& -\int_{0}^{2 \pi} d \varphi \int_{-\infty}^{+\infty}\left(d^{2} \overrightarrow{\mathbf{y}}\right) f(\overrightarrow{\mathbf{y}}) \frac{\mathcal{P}}{\left[\left\langle\overrightarrow{\mathbf{n}_{\varphi}},(\overrightarrow{\mathbf{x}}-\overrightarrow{\mathbf{y}})\right\rangle\right]^{2}}
\end{aligned}
$$

Hence, it becomes clear that the first term in Eq. (16) merely disappears when we split the angular integration into two parts,

$$
\int_{0}^{2 \pi} d \varphi=\int_{0}^{\pi} d \varphi+\int_{\pi}^{2 \pi} d \varphi
$$

It is instructive to present an alternative way to derive the inverse Radon transform. With the help of (18) and after the replacements given by $\varphi=\phi+\pi$ and $\lambda=-\tilde{\lambda}$, Eq. (12) takes the following form:

$f(\overrightarrow{\mathbf{x}})=\int_{0}^{\pi} d \varphi \int_{-\infty}^{+\infty} d \lambda \lambda \varepsilon(\lambda) e^{+i \lambda\left\langle\overrightarrow{\mathbf{n}}_{\varphi}, \overrightarrow{\mathbf{x}}\right\rangle} \mathcal{F}[f](\lambda, \varphi)$.

We now use the integral representation of the $\varepsilon$-function,

$$
\varepsilon(\lambda)=\frac{1}{\pi i} \int_{-\infty}^{+\infty} d \rho e^{+i \lambda \rho} \frac{\mathcal{P}}{\rho}
$$

and perform two consecutive replacements: the first replacement $\rho+\left\langle\overrightarrow{\mathbf{n}}_{\varphi}, \overrightarrow{\mathbf{x}}\right\rangle=\eta$ has to be implemented just after inserting (20) into (19) and the second replacement $\tau-\eta=\tilde{\tau}$ after using the representation (4) in Eq. (19). Hence, we have

$$
\begin{aligned}
f(\overrightarrow{\mathbf{x}})= & 2 \int_{0}^{\pi} d \varphi \int_{-\infty}^{+\infty}(d \eta) \frac{\mathcal{P}}{\eta-\left\langle\overrightarrow{\mathbf{n}}_{\varphi}, \overrightarrow{\mathbf{x}}\right\rangle} \\
& \times \int_{-\infty}^{+\infty} d \tau \mathcal{R}[f](\eta+\tau, \varphi) \frac{\partial}{\partial \tau} \delta(\tau)
\end{aligned}
$$

and after some simple algebra we get

$f(\overrightarrow{\mathbf{x}})=-2 \int_{0}^{\pi} d \varphi \int_{-\infty}^{+\infty}(d \eta) \frac{\mathcal{P}}{\left(\eta-\left\langle\overrightarrow{\mathbf{n}}_{\varphi}, \overrightarrow{\mathbf{x}}\right\rangle\right)^{2}} \mathcal{R}[f](\eta, \varphi)$.

Comparing Eqs. (16) and (22), one can see that if, in Eq. (16), we split the angular integration as in Eq. (18), after the consecutive replacements of integration variables we reproduce Eq. (22). It means that the first additional term of Eq. (16) is an insubstantial contribution if we have the full angular integration in the interval $[0,2 \pi]$ and the symmetric integration measure of the radial $\eta$-parameter.

It is worth noticing that the representation (22) fully coincides with the result that can be found in $[14,15]$ where the integration over the full angular region, $\varphi \in[0,2 \pi]$, has been used a priori.

However, as we demonstrate in Sec. II B, there are some cases where for the restricted support of $f$ the angular parameter $\varphi$ together with the radial parameter $\eta$ meet the corresponding restrictions limiting the variation intervals and breaking the symmetry condition generated by Eq. (10). At the same time, as explained in Sec. III, in the case of the generalized transverse-momentum dependent distributions it turns out that the angular integration is also limited by the region $\varphi \in[-\pi / 2, \pi / 2]$ only owing to the fact that the $T$-reversal invariance has been relaxed. Hence, we deal with the situation where there is no the symmetry presented by (10) as well. As a result, it is important for the first additional term of Eq. (16) to be included in the analysis.

\section{B. The case of restricted support of function $f$}

In the preceding subsection, we have considered the simplest case of unbounded support of function $f$. However, in any practical applications of the Radon transforms, as a rule the support of function $f$ is restricted by some region. In this subsection, we study the influences of the restricted support on the Fourier and Radon transformations. Namely, we demonstrate that, in the most general case, the restricted support of $f$ leads to the 
restrictions in the $q$-plane where the Fourier image of $f$ has been determined.

To begin with, let us return to the Fourier slice theorem; see Eq. (4). Notice that the integral representation of unit (2), which has been inserted into the direct Fourier transform (1), can actually be presented in different forms. Indeed, we have

$$
\begin{aligned}
& \int_{-\infty}^{+\infty}(d t) \delta(t-\langle\overrightarrow{\mathbf{q}}, \overrightarrow{\mathbf{x}}\rangle)=\int_{-\infty}^{+\infty}(d \tau) \delta\left(\tau-\left\langle\overrightarrow{\mathbf{n}}_{\varphi}, \overrightarrow{\mathbf{x}}\right\rangle\right) \\
& =\int_{-\infty}^{+\infty}(d z) \delta\left(z-x_{1}-\xi x_{2}\right)=1,
\end{aligned}
$$

where

$$
\begin{aligned}
& z=\frac{t}{q_{1}} \quad \text { with } \quad d z=\frac{d t}{q_{1}}, \\
& \xi=\frac{q_{2}}{q_{1}} .
\end{aligned}
$$

Then, we write down the direct Fourier transform as

$$
\begin{aligned}
\mathcal{F} & {[f]\left(q_{1}, q_{2}\right) } \\
& =\left.\int_{-\infty}^{+\infty} d^{2} \overrightarrow{\mathbf{x}} e^{-i\langle\overrightarrow{\mathbf{q}}, \overrightarrow{\mathbf{x}}\rangle} f(\overrightarrow{\mathbf{x}}) \int_{-\infty}^{+\infty}(d z) \delta\left(z-x_{1}-\xi x_{2}\right)\right|_{\xi=\frac{q_{2}}{q_{1}}} \\
& =\left.\int_{-\infty}^{+\infty}(d z) e^{-i q_{1} z}\left\{\int_{-\infty}^{+\infty} d^{2} \overrightarrow{\mathbf{x}} f(\overrightarrow{\mathbf{x}}) \delta\left(z-x_{1}-\xi x_{2}\right)\right\}\right|_{\xi=\frac{q_{2}}{q_{1}}} .
\end{aligned}
$$

Hence, the Fourier slice theorem takes now the following form [16]:

$$
\int_{-\infty}^{+\infty}(d z) e^{-i q_{1} z} \mathcal{R}[f](z, \xi)=\left.\mathcal{F}[f]\left(q_{1}, \xi q_{1}\right)\right|_{\xi=\frac{q_{2}}{q_{1}}} .
$$

In contrast to Eq. (4), this representation deals with $\left(q_{1}, \xi\right)$ as an independent set of variables for the Fourier transform. As a result, the $\delta$-function in the direct Radon transform, $\delta\left(z-x_{1}-\xi x_{2}\right)$, gives $\left(z, \xi, x_{2}\right)$ as the independent variables, i.e.,

$$
\mathcal{R}[f](z, \xi)=\int_{-\infty}^{+\infty} d x_{2} f\left(z-\xi x_{2}, x_{2}\right)
$$

So far, we did not impose any support restrictions for the function $f$. Now, for the sake of simplicity, let $\overrightarrow{\mathbf{x}}$ be in the region $[-1,1]$, i.e., $\overrightarrow{\mathbf{x}} \in \Omega_{\square}$, and let $f$ be a homogeneous function. Regarding the function support, this example is very close to the situation that appears in the case of GPDs.

We want to show that the support restrictions applied for the function $f$ lead to the corresponding restrictions for the support of function $\mathcal{F}[f](\overrightarrow{\mathbf{q}})$ in $q$-plane and, therefore, to the restrictions for the (inverse) Radon transformations; see Eq. (4).

Owing to $z-x_{1}-\xi x_{2}=0$, which is an argument of the $\delta$-function in the Radon transform (26), one can readily derive that

$$
\text { if } x_{1} \in[-1,1], \quad \text { then } z+1 \geq \xi x_{2} \geq z-1 \text {. }
$$

Since $x_{2} \in[-1,1]$, the given inequality generates four different inequalities depending on the position of $(z \pm$ $1) / \xi$ in the interval $[-1,1]$, i.e.,

$$
\begin{aligned}
& \text { (A): } \frac{z+1}{\xi} \quad \text { and } \quad \frac{z-1}{\xi} \in[-1,1] ; \\
& (B): \frac{z+1}{\xi} \in[-1,1], \quad \frac{z-1}{\xi} \notin[-1,1] ; \\
& (C): \frac{z+1}{\xi} \notin[-1,1], \quad \frac{z-1}{\xi} \in[-1,1] ; \\
& (D): \frac{z+1}{\xi} \notin[-1,1], \quad \frac{z-1}{\xi} \notin[-1,1] .
\end{aligned}
$$

However, for our demonstration, it is enough to consider the case $(A)$. So, we dwell on the following inequalities:

$$
1 \geq \frac{\mathrm{a}+1}{\xi} \geq x_{2} \geq \frac{z-1}{\xi} \geq-1 .
$$

Since we want to study the support restrictions in the Cartesian system, the next steps are the following: (a) we replace the angular (slope) parameter $\xi$ in (34) by the Cartesian variables $\left(q_{1}, q_{2}\right)$ using Eq. (25); (b) we consider $q_{2}$ as a function of $q_{1}$ and $z$ (here, $z$ plays a role of an external parameter for this kind of function) in $q$-plane.

For our convenience, we also introduce the following regions in $q$-plane:

$$
\begin{aligned}
\Omega^{I}:\left\{q_{2}>0, q_{1}>0\right\} & \doteq \overrightarrow{\mathbf{q}}^{I} ; \\
\Omega^{I I}:\left\{q_{2}<0, q_{1}>0\right\} & \doteq \overrightarrow{\mathbf{q}}^{I I} ; \\
\Omega^{I I I}:\left\{q_{2}<0, q_{1}<0\right\} & \doteq \overrightarrow{\mathbf{q}}^{I I I} \\
\Omega^{I V}:\left\{q_{2}>0, q_{1}<0\right\} & \doteq \overrightarrow{\mathbf{q}}^{I V} .
\end{aligned}
$$

Given that, for the definiteness, we assume $\xi$ and $z+1$ to be the positive values, i.e., $\{\xi, z+1\}>0$. Hence, the condition $a$ of (34) together with (25) give us that

$$
(z+1) q_{1}^{I} \leq q_{2}^{I} \quad \text { and } \quad(z+1) q_{1}^{I I I} \geq q_{2}^{I I I} .
$$

At the same time, the case of the negative values for $\xi$ and $z+1$ corresponds to the following conditions: 


$$
|z+1| q_{1}^{I I} \leq-q_{2}^{I I} \quad \text { and } \quad-|z+1| q_{1}^{I V} \leq q_{2}^{I V} .
$$

We emphasize that the equalities in (36) and (37) like $(z+1) q_{1}^{I}=q_{2}^{I}$ etc., correspond finally to the requirement that reduces a number of independent variables. Since, from the very beginning, we have considered $f$ as a function of two independent variables, the boundaries defined by the equalities in (36) and (37) should be excluded from the domain.

In particular cases, these statements are known as the following theorem in the mathematical literature: if $f$ is a function of $n$ independent variables, then the Radon transform of $f, \mathcal{R}[f]$, depends on $n$ independent variables too. That is, the Radon transform is a bijection and lives on $\mathbb{R}^{1} \times S^{n-1}[14]$.

To conclude, the inequalities (without the equalities) of (36) and (37) define the so-called physical domain depicted in Fig. 1 where the two-dimensional Fourier and, therefore, the Radon transforms have been uniquely determined for the function $f$ with the restricted support: $\operatorname{supp}(f)=\Omega_{\square}$. This ultimately means that the function $f$ can be reconstructed by the inverse Radon transform if the support of Fourier function $\mathcal{F}[f](\overrightarrow{\mathbf{q}})$ in $q$-plane has been compactly distributed along the $q_{2}$ axis within the right and/or left $\Omega$-regions; see Fig. 1 .

In other words, the correct inverse Radon transform should be implemented with the angular parameter $\varphi$, which is limited by the region $[0, \pi]$ (or, after shifting, by $[-\pi / 2, \pi / 2]$ ) corresponding to Fig. 1 . This allows the first term in Eq. (16) to exist and to contribute to the inversion of Radon transform in the case of $f(\overrightarrow{\mathbf{x}}) \in \mathbb{C}$.

\section{The case of localized support of function $f$}

The same conclusions on the restrictions in the Cartesian system can be demonstrated by the direct calculation of the Radon image of the simple localized function. Indeed, if we suppose that the localized function has been defined as (here, $\overrightarrow{\mathbf{A}}$ is an arbitrary external vector)

$$
f(\overrightarrow{\mathbf{x}} \mid \overrightarrow{\mathbf{A}})=e^{-(\overrightarrow{\mathbf{x}}-\overrightarrow{\mathbf{A}})^{2}}
$$

the direct Radon image of this function reads

$$
\mathcal{R}[f](\tau, \varphi \mid \overrightarrow{\mathbf{A}})=\int_{-\infty}^{+\infty} d^{2} \overrightarrow{\mathbf{x}} e^{-(\overrightarrow{\mathbf{x}}-\overrightarrow{\mathbf{A}})^{2}} \delta\left(\tau-\left\langle\overrightarrow{\mathbf{n}}_{\varphi}, \overrightarrow{\mathbf{x}}\right\rangle\right)
$$

After direct calculations, we get (here, $C$ is a constant)

$$
\mathcal{R}[f]\left(\tau-\left\langle\overrightarrow{\mathbf{n}}_{\varphi}, \overrightarrow{\mathbf{A}}\right\rangle, \varphi\right)=C e^{-\left(\tau-\left\langle\overrightarrow{\mathbf{n}}_{\varphi}, \overrightarrow{\mathbf{A}}\right\rangle\right)^{2}} .
$$

Indeed, after the replacement $\overrightarrow{\mathbf{y}}=\overrightarrow{\mathbf{x}}-\overrightarrow{\mathbf{A}}$ in Eq. (39), which leads to

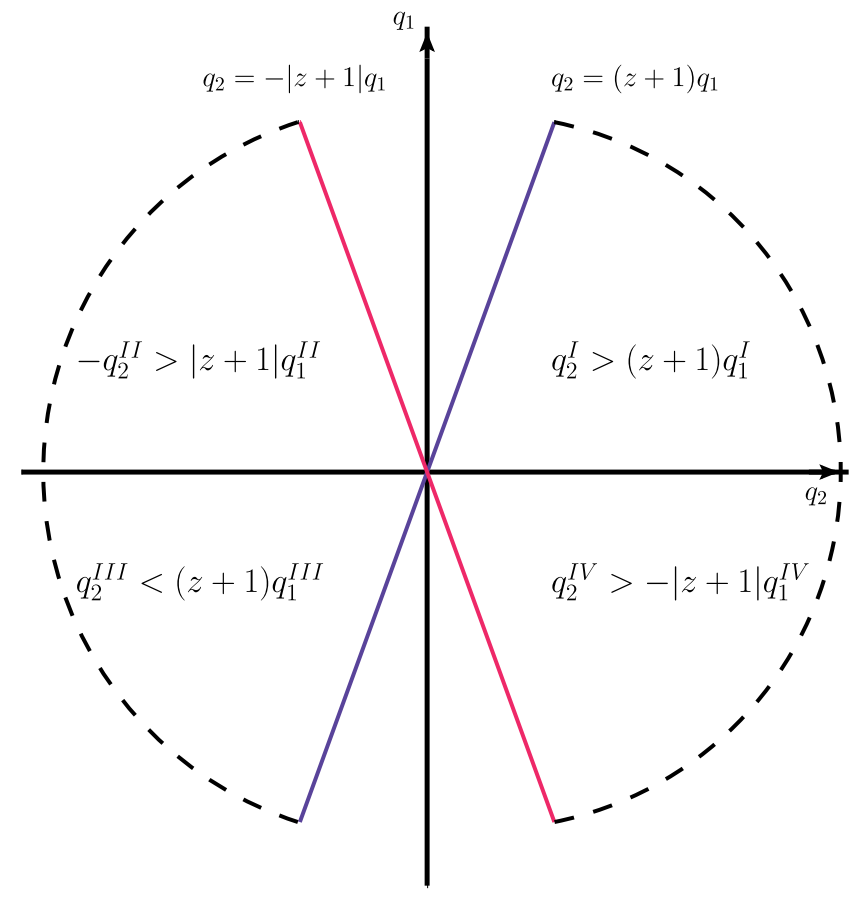

FIG. 1. The $\Omega$-regions in $q$-plane for the Fourier function support.

$$
\begin{aligned}
\mathcal{R}[f](\tau, \varphi \mid \overrightarrow{\mathbf{A}}) & =\int_{-\infty}^{+\infty} d^{2} \overrightarrow{\mathbf{y}} e^{-\overrightarrow{\mathbf{y}}^{2}} \delta\left(\tau-\left\langle\overrightarrow{\mathbf{n}}_{\varphi}, \overrightarrow{\mathbf{A}}\right\rangle-\left\langle\overrightarrow{\mathbf{n}}_{\varphi}, \overrightarrow{\mathbf{y}}\right\rangle\right) \\
& =\mathcal{R}[f]\left(\tau-\left\langle\overrightarrow{\mathbf{n}}_{\varphi}, \overrightarrow{\mathbf{A}}\right\rangle, \varphi\right) \equiv \mathcal{R}[f](\tilde{\tau}, \varphi),
\end{aligned}
$$

we perform the rotation of the system, see Eq. (6), to obtain the following representation:

$$
\begin{aligned}
\mathcal{R}[f](\tilde{\tau}, \varphi) & =\int_{-\infty}^{+\infty} d p d s e^{-p^{2}-s^{2}} \delta(\tilde{\tau}-p) \\
& =e^{-\tilde{\tau}^{2}} \int_{-\infty}^{+\infty} d s e^{-s^{2}}=C e^{-\left(\tau-\left\langle\overrightarrow{\mathbf{n}}_{\varphi}, \overrightarrow{\mathbf{A}}\right\rangle\right)^{2}} .
\end{aligned}
$$

Then, we replace the polar Radon coordinates $(\tau, \varphi)$ by the Cartesian coordinates $(u, v)$ as

$$
\begin{aligned}
\tau & =\sqrt{\overrightarrow{\mathbf{K}}^{2}}, & \overrightarrow{\mathbf{K}} & =(u, v), \\
u & =\tau \cos \varphi, & v & =\tau \sin \varphi .
\end{aligned}
$$

With these, we derive that

$$
\mathcal{R}[f](u, v \mid \overrightarrow{\mathbf{A}})=C e^{-\frac{\overrightarrow{\mathbf{K}}^{2}-\langle\overrightarrow{\mathbf{K}} \cdot \overrightarrow{\mathbf{A}}\rangle}{\sqrt{\overrightarrow{\mathbf{K}}^{2}}}},
$$

which is localized in the Cartesian system of coordinates $(u, v)$. 


\section{THE RADON TRANSFORMS FOR GPDS AND DD FUNCTIONS: THE SUPPORT THEOREM AND THE EXTRA GPDS PROPERTIES}

As stated above, we have considered the (inverse) Radon transforms defined for an arbitrary functions $f$ with the unbounded and compact supports. We have demonstrated that the restricted support of $f$ defined as $\operatorname{supp}(f)=\Omega_{\square}$ leads to the restriction imposed on the angular Radon parameter $\varphi \in[-\pi / 2, \pi / 2]$. Now, based on the results presented in the preceding sections, we overhaul the (inverse) Radon transforms, which relate the GPDs to DDs (cf. [8]). Since, as is well known, the support of DD function is similar to the considered region $\Omega_{\square}$ (modulo the corresponding rotation and rescaling), we should expect that a similar restriction on the angular Radon parameter takes place also in the case of GPDs independently of the presence of the primordial transverse momenta. We recall that the $k_{\perp}$-dependence of the parton distributions results in the relaxing of $T$-reversal invariance and, therefore, the DD functions for the positive and negative variable $x_{2}$ (or, in the standard notation, $\beta$, see Fig. 2) start to be independent ones; see Sec. IV.

In the first part of this section, we show that the restricted support of the DD function in the physical domain can conditionally lead to the physical restrictions for the GPD variables $z$ and $\xi$. In contrast to the abstract mathematical case considered in the preceding section, we deal now with the physical interpretation of the angular Radon parameter $\xi$. Namely, we have both the physical, $\xi \leq 1$

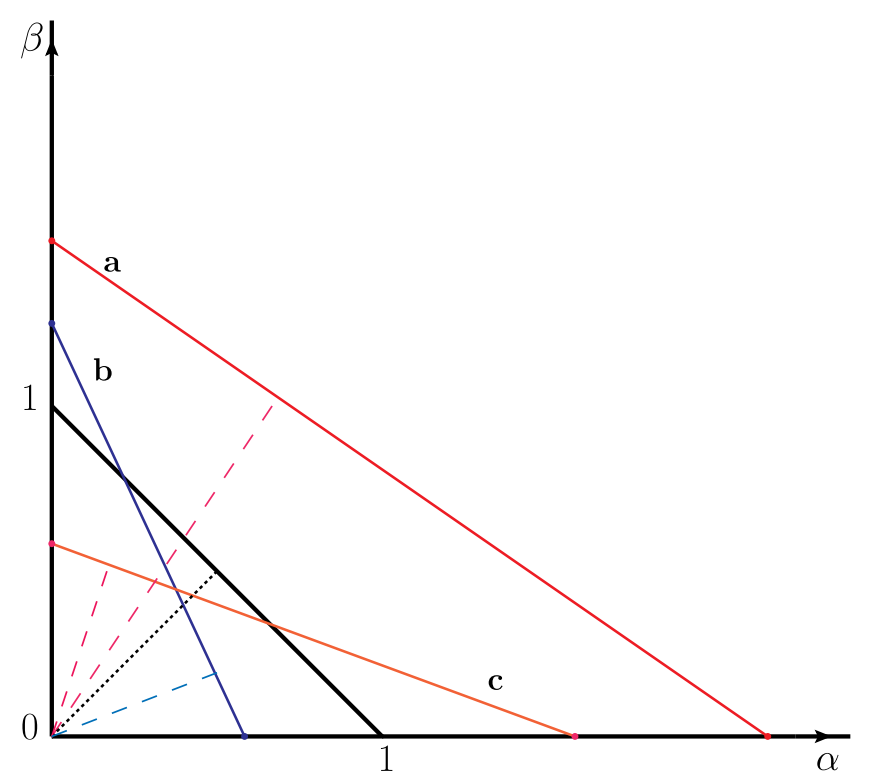

FIG. 2. The $\bar{\Omega}^{I}$ region of the DD-function support and the three classes of lines, $\beta=z / \xi-\alpha / \xi$, related to the corresponding Radon transforms. Notations are as follows: a: $\Rightarrow$ $\{z / \xi>1($ or $z>\xi)\} \bigcup\{z>1, \xi>1($ or $\varphi>\pi / 4)\} ; \mathrm{b}: \Rightarrow\{z / \xi>1$ $($ or $z>\xi)\} \bigcup\{z<1, \xi<1($ or $\varphi<\pi / 4)\} ; \quad \mathrm{c}: \Rightarrow\{z / \xi<1$ $($ or $z<\xi)\} \bigcup\{z>1, \xi>1($ or $\varphi>\pi / 4)\}$. (this is the GPDs region), and unphysical, $\xi>1$ (this is the GDA region), values of the angular Radon parameter.

In the second part of the section, we study the extra properties of GPDs that come from the requirement of $h_{A}=0$.

\section{A. The support theorem}

We begin with the standard relation between GPDs and DDs in the following form [3],

$$
H(z, \xi)=\int_{-\infty}^{+\infty} d \alpha d \beta \tilde{h}(\alpha, \beta) \delta(z-\alpha-\xi \beta),
$$

where

$$
\begin{aligned}
\tilde{h}(\alpha, \beta) & =h(\alpha, \beta) \Theta(\{\alpha, \beta\} \in \bar{\Omega}), \\
\int_{\bar{\Omega}} d \alpha d \beta & \doteq \int_{-1}^{+1} d \alpha \int_{-1+|\alpha|}^{1-|\alpha|} d \beta
\end{aligned}
$$

As usual, the function $h(\alpha, \beta)$ involves the standard DD functions $f(\alpha, \beta)$ and $g(\alpha, \beta)$ [7], but it is irrelevant now.

The full support region $\bar{\Omega}$, which is a symmetric rhombus, can be split up into four subregions, $\bar{\Omega}^{I}, \ldots, \bar{\Omega}^{I V}$, where, for example, the first region $\bar{\Omega}^{I}$ is given by $0 \leq \alpha+\beta \leq 1$ together with $0 \leq \alpha \leq 1$ and $0 \leq \beta \leq 1$ and in the similar manner the other regions can be determined.

Let us write Eq. (5) in the equivalent form as

$$
\begin{aligned}
& \mathcal{R}[f](\eta, \varphi)|\cos \varphi| \\
& \quad=\int_{\bar{\Omega}} d x_{1} d x_{2} f\left(x_{1}, x_{2}\right) \delta\left(\frac{\eta}{\cos \varphi}-x_{1}-x_{2} \tan \varphi\right)
\end{aligned}
$$

and, as above, we introduce the following relations:

$$
\begin{gathered}
\mathcal{R}[f](\eta, \varphi)|\cos \varphi| \Rightarrow H\left(\frac{\eta}{\cos \varphi}, \tan \varphi\right)=H(z, \xi) \\
\frac{\eta}{\cos \varphi} \Rightarrow z, \quad \tan \varphi \Rightarrow \xi, \quad f\left(x_{1}, x_{2}\right) \Rightarrow h(\alpha, \beta) .
\end{gathered}
$$

As shown in Sec. II, the restricted support of any arbitrary function [e.g., $\tilde{h}(\alpha, \beta)$ ] induces the maximal angular restriction $\varphi \in[-\pi / 2, \pi / 2]$ (or $\xi \in[-\infty, \infty]$ ), where the symmetry property presented by Eq. (10) has been broken leaving the first term in Eq. (16) contributing; see Sec. IV.

Let us be limited by consideration of the region $\bar{\Omega}^{I}$, which is enough in most cases. If the support of $h(\alpha, \beta)$ is fixed and defined by $\bar{\Omega}^{I}$, then a question that has arisen is that whether the support of the $H$-function in Eq. (45) is automatically giving the region $0 \leq\{z, \xi\} \leq 1$. To cover the region $\bar{\Omega}^{I}$, the Radon parameters $\eta$ and $\varphi$ have to vary within the intervals $[0,1]$ and $[0, \pi / 2]$, respectively. Hence, at the moment we deal with the unbounded region in terms of $z$ and $\xi$, i.e., $\{z, \xi\} \in[0,+\infty]$. 
On the other hand, making use of the integral representation of the $\Theta$-function defined as

$$
\begin{aligned}
& \Theta\left(\{\alpha, \beta\} \in \bar{\Omega}^{I}\right) \\
& \quad \equiv \theta(0 \leq \alpha+\beta \leq 1) \theta(0 \leq \alpha \leq 1) \theta(0 \leq \beta \leq 1) \\
& \quad=\left.\int_{0}^{1} d T \delta(T-\alpha-\beta)\right|_{0 \leq\{\alpha, \beta\} \leq 1},
\end{aligned}
$$

we obtain that the rhs of (45) reads

$$
\int_{0}^{1} d T \int_{-\infty}^{+\infty} d \alpha d \beta h(\alpha, \beta) \delta(T-\alpha-\beta) \delta(z-\alpha-\xi \beta) .
$$

After integrating over $d \alpha$ and $d \beta$ with two $\delta$-functions, we derive that

$$
H(z, \xi)=\frac{1}{|1-\xi|} \int_{0}^{1} d T h\left(\frac{z-\xi T}{1-\xi}, \frac{T-z}{1-\xi}\right)
$$

provided

$$
0 \stackrel{\mathrm{a}}{\leq} \frac{z-\xi T}{1-\xi} \stackrel{\mathrm{b}}{\leq} 1, \quad 0 \stackrel{\mathrm{c}}{\leq} \frac{T-z}{1-\xi} \stackrel{\mathrm{d}}{\leq} 1 .
$$

Considering the condition $a$ of (52), we have

$$
\begin{aligned}
& \text { if } 1-\xi \geq 0, \quad \text { then } z \geq \xi T, \\
& \text { if } 1-\xi \leq 0, \quad \text { then } z \leq \xi T .
\end{aligned}
$$

Hence, it means that $z, \xi$ can be unbounded. At the same time, focusing on the condition $b$ of (52), we have

$$
\begin{aligned}
& \text { if } 1-\xi \geq 0, \quad \text { then } \xi(T-1) \geq z-1 \\
& \Rightarrow z \leq 1 \quad \text { or } \quad z \leq 1+\xi(T-1), \\
& \text { if } 1-\xi \leq 0, \quad \text { then } \xi(T-1) \leq z-1 \\
& \Rightarrow z \geq 1 \quad \text { or } \quad z \geq 1+\xi(T-1) .
\end{aligned}
$$

In the same manner, we are able to analyze the conditions $c$ and $d$ of (52). From the condition $c$ of (52), we obtain that

$$
\begin{aligned}
& \text { if } 1-\xi \geq 0, \quad \text { then } T \geq z \Rightarrow z \leq 1, \\
& \text { if } 1-\xi \leq 0, \quad \text { then } T \leq z,
\end{aligned}
$$

and the condition $d$ of (52) gives us the following:

$$
\begin{aligned}
& \text { if } 1-\xi \geq 0, \quad \text { then } T+\xi \leq 1+z, \\
& \text { if } 1-\xi \leq 0, \quad \text { then } T+\xi \geq 1+z .
\end{aligned}
$$

Thus, we can infer that the conditions (52) cannot ensure, generally speaking, the physical restrictions for the parameters $z$ and $\xi$ unless we impose $\xi \leq 1$ from the very beginning. Figure 2 demonstrates graphically the above statement and shows the three most useful classes of the lines. At the same time, this figure reflects the well-known situation that can be presented as

$$
\begin{gathered}
H^{\mathrm{GPD}}(z, \xi) \theta(\xi \leq 1) \oplus H^{\mathrm{GDA}}(z, \xi) \theta(\xi>1) \\
=\int_{-\infty}^{+\infty} d \alpha d \beta \tilde{h}(\alpha, \beta) \delta(z-\alpha-\xi \beta) .
\end{gathered}
$$

We can see that the line denoted as a corresponds to the unphysical regions for $z$ and $\xi$ and, according to the Boman-Quinto theorem [17], this class of lines can be excluded from consideration. Line $\mathbf{b}$ in Fig. 2 corresponds to the physical region for $z$ and $\xi$ (describing, in this case, the DGLAP region for the GPDs), while line c corresponds to the unphysical region of parameters $z$ and $\xi$ and describes the GDA.

Hence, to reconstruct the DD function by the inverse Radon transforms we are forced to deal with the $\mathrm{H}$ function, which is supported by the unphysical region $\{\xi>1, z \in \forall\}$ [8]. In other words, for the $t$ channel, the physical region of the $h$-function should also require our knowledge of the physical region of the $H$-function in the $s$-channel. This is one of exhibitions of the ill-posed problem related to the inverse Radon transforms $[9,18]$. The other formulation of ill-posed problem has been presented in the Appendix A.

\section{B. The additional term $f_{A}$}

We are now in position to study the properties of the additional term $f_{A}$ (or $h_{A}$ ). As follows from Sec. II, if we have the angular parameter $\varphi$ that varies in the full interval between 0 and $2 \pi$, then we can conclude that

$$
\begin{aligned}
\frac{i}{\pi} f_{A}(\overrightarrow{\mathbf{x}})= & \int_{-\infty}^{+\infty}(d \eta) \delta(\eta) \int_{0}^{2 \pi} d \varphi \\
& \times\left[\frac{\partial}{\partial \eta} \mathcal{R}[f]\left(\eta+\left\langle\overrightarrow{\mathbf{n}}_{\varphi}, \overrightarrow{\mathbf{x}}\right\rangle, \varphi\right)\right]=0 .
\end{aligned}
$$

We can also treat this equation as the condition that GPDs have to fulfil. In other words, in order to satisfy Eq. (58) the corresponding GPDs should have definite symmetric properties. Indeed, since the skewness parameter $\xi$ is determined by $\tan \varphi$ [let us first make a replacement given by $\tilde{\varphi}=\varphi-\pi / 2$ in the integrand of Eq. (58)], we obtain

$$
\begin{aligned}
\frac{i}{\pi} f_{A}(\overrightarrow{\mathbf{x}})= & \int_{-\infty}^{+\infty}(d \eta) \delta(\eta)\left[\int_{-\frac{\pi}{2}}^{\frac{\pi}{2}} d \tilde{\varphi}+\int_{\frac{\pi}{2}}^{\frac{3 \pi}{2}} d \tilde{\varphi}\right] \\
& \times\left[\frac{\partial}{\partial \eta} \mathcal{R}[f]\left(\eta+\left\langle\overrightarrow{\mathbf{n}}_{\tilde{\varphi}+\frac{\pi}{2}}, \overrightarrow{\mathbf{x}}\right\rangle, \tilde{\varphi}+\frac{\pi}{2}\right)\right]=0 .
\end{aligned}
$$

Here, the Radon transform in the integrand can be presented as 


$$
\begin{aligned}
\mathcal{R} & {[f]\left(\eta+\left\langle\overrightarrow{\mathbf{n}}_{\tilde{\varphi}+\frac{\pi}{2}}, \overrightarrow{\mathbf{x}}\right\rangle, \tilde{\varphi}+\frac{\pi}{2}\right) } \\
& =\int_{-\infty}^{+\infty} d^{2} \overrightarrow{\mathbf{y}} f\left(y_{1}, y_{2}\right) \delta\left(\eta-\left(x_{1}-y_{1}\right) \sin \tilde{\varphi}+\left(x_{2}-y_{2}\right) \cos \tilde{\varphi}\right) \\
& =\int_{-\infty}^{+\infty} d^{2} \overrightarrow{\mathbf{y}} f\left(y_{2}, y_{1}\right) \delta\left(\eta-\left(x_{1}-y_{2}\right) \sin \tilde{\varphi}+\left(x_{2}-y_{1}\right) \cos \tilde{\varphi}\right) \\
& =\int_{-\infty}^{+\infty} d^{2} \overrightarrow{\mathbf{y}} \frac{f\left(y_{2}, y_{1}\right)}{|\cos \tilde{\varphi}|} \delta\left(\eta+x_{2}-\xi x_{1}-y_{1}+\xi y_{2}\right)
\end{aligned}
$$

Given that, using Eqs. (47) and (48) we readily derive that

$\frac{\partial}{\partial x_{2}} \int_{-\infty}^{\infty} d \xi\left\{\bar{H}\left(x_{2}-\xi x_{1},-\xi\right)+\bar{H}\left(x_{2}+\xi x_{1},+\xi\right)\right\}=0$,

where a new function $\bar{H}$ is introduced to be equal to

$$
\bar{H}(z, \xi)=\int_{-\infty}^{+\infty} d^{2} \overrightarrow{\mathbf{y}} f\left(y_{2}, y_{1}\right) \delta\left(z-y_{1}-\xi y_{2}\right) .
$$

To obtain Eq. (61), the derivative over $d \eta$ in Eq. (59) has been replaced by the derivative over $d x_{2}$ and the integration over $d \eta$ has been performed. We stress that in contrast to the standard function $H$, see Eq. (45), the function $\bar{H}$ introduced in Eq. (62) corresponds to the Radon transform of $f\left(y_{2}, y_{1}\right)$ rather than $f\left(y_{1}, y_{2}\right)$.

Therefore, to fulfil the condition (61), the GPDs have to obey either the symmetry given by

$$
\bar{H}\left(x_{2}-\xi x_{1},-\xi\right)=-\bar{H}\left(x_{2}+\xi x_{1}, \xi\right)
$$

or

$$
\frac{\partial}{\partial x_{2}} \bar{H}\left(x_{2} \pm \xi x_{1}, \pm \xi\right)=0
$$

The condition (64) cannot to be realized because the function $f_{A}$ is a function of two variables by construction. With respect to the symmetry defined by (63), it can be considered as the symmetry properties for the standard GPDs $H$ under the time-reverse transforms provided that the function $f$ satisfies the requirement

$$
f\left(y_{1}, y_{2}\right)= \pm f\left(y_{2}, y_{1}\right) .
$$

Notice that if we deal with the case where the timereverse invariance is so relaxed that we have no such GPDs that obey the condition (63), then the additional term $f_{A}$ exists.

\section{ADDITIONAL TERM $h_{A}$ AND GENERALIZED TRANSVERSE-MOMENTUM DEPENDENT FUNCTIONS}

In this section, we give a possible physical interpretation of the additional term $f_{A}$ discussed in the previous sections.
Since one of the important features of $f_{A}$ is its complexness, our interpretation is based on the generalized transverse-momentum dependent functions (GTMD functions), where the imaginary parts of GTMD functions exist due to the fact that the time-reversal invariance has been relaxed (below, we give the exact definition of the time-reversal relaxing). To this end, we also introduce the DD functions, which involve the transverse-momentum $k_{\perp}$ dependence.

We begin with the momentum dependent functions that can be introduced as (within the Heisenberg representation)

$W_{s_{1} s_{2}}^{[\Gamma]}(k ; \bar{P}, \Delta)=\int\left(d^{4} z\right) e^{+i K \cdot z}\left\langle p_{2}, s_{2}\left|\mathcal{O}^{[\Gamma]}(0 ; z)\right| p_{1}, s_{1}\right\rangle$,

where

$$
\begin{aligned}
& \mathcal{O}^{[\Gamma]}(0 ; z)=: \bar{\psi}(0) \Gamma[0 ; z]_{A} \psi(z): \\
& K=k-\frac{\Delta}{2}, \quad \bar{P}=\frac{p_{2}+p_{1}}{2}, \\
& \Delta=p_{2}-p_{1}, \quad-2 \xi=\frac{\Delta^{+}}{\bar{P}^{+}} .
\end{aligned}
$$

We now go over to the so-called fractional projections that appear as a result of factorization; we have

$$
\begin{aligned}
& W_{s_{1} s_{2}}^{[\Gamma]}\left(x, \overrightarrow{\mathbf{k}}_{\perp} ; \bar{P}, \Delta\right) \\
& \quad=\int\left(d k^{-}\right)\left(d k^{+}\right) \delta\left(x-k^{+} / \bar{P}^{+}\right) W_{s_{1} s_{2}}^{[\Gamma]}(k ; \bar{P}, \Delta) \\
& \quad=\int\left(d z^{-}\right)\left(d^{2} \overrightarrow{\mathbf{z}}_{\perp}\right) e^{+i K^{+} z^{-}-i \overrightarrow{\mathbf{k}}_{\perp} \overrightarrow{\mathbf{z}}_{\perp}}\left\langle p_{2}, s_{2}\left|\mathcal{O}^{[\Gamma]}(0 ; z)\right| p_{1}, s_{1}\right\rangle .
\end{aligned}
$$

In the $k_{\perp}$-independent $\left(k_{\perp}\right.$-integrated) case, there is no a doubt that we deal with the time-reversal and parity invariance that, together with the hermiticity, ultimately lead to the restricting conditions for the possible forms of parametrizations through the different kinds of parton distributions (GPDs/GDAs, PDF, etc.) and the corresponding Lorentz covariant tensors. As a result, all of the parton distributions, for example GPDs, possess the well-known symmetry properties and are the real functions [7]. However, if the transverse momenta $k_{\perp}$ remain unintegrated, see Eq. (69), the time-reversal invariance has been relaxed in a sense that in the Lorentz covariant parametrization of the hadron matrix elements the time-reversal transforms do not impose any constraints on the parametrizing functions and consequently allow the existence of the $T$-odd parton distributions [19-21]. Moreover, such parton distributions as GTMDs become complex functions [20,21].

Indeed, for the transverse-momentum dependent functions, which take part in the parametrization of the hadron matrix elements of the gauge-invariant operators, the time-reversal transforms lead to the following relation: 


$$
\left\{W_{[ \pm]}^{[\Gamma]}\left(x, \overrightarrow{\mathbf{k}}_{\perp} ; \bar{P}, \Delta\right)\right\}^{*}=(-1)^{\eta} W_{[\mp]}^{\left[\pi \Gamma^{*} \mathbb{T}\right]}(x, \tilde{\overrightarrow{\mathbf{k}}} \perp ; \tilde{\bar{P}}, \tilde{\Delta}),
$$

where $\tilde{a}=\left(a_{0},-\vec{a}\right), \mathbb{T}=i \gamma^{1} \gamma^{3}=-i \gamma_{5} C, \eta$ defines the parity of transformations $(\eta=1$ for the odd combinations, while $\eta=2$ for the even combinations), and $[ \pm]$ implies the future-pointed and past-pointed Wilson line in the corresponding correlators. For example, the nucleon matrix elements of the nonlocal quark operators yield to the relation (70) with $\eta=2$ for all $\Gamma$ of the Dirac $\gamma$ basis $[20,21]$. It is important to emphasize that the $k_{\perp}$-dependent parton functions are actually the complex functions owing to the fact that the future-pointed Wilson line is converting to the past-pointed Wilson line under the time-reversal transforms [19].

We dwell on the nucleon GTMDs that are generated by $\gamma^{+}$-projection. Some of them can be presented as

$$
\begin{aligned}
& W^{\left[\gamma^{+}\right]}(k ; \bar{P}, \Delta) \\
& =\bar{u}\left(p_{2}\right)\left[H\left(x, \xi, t ;\left\{\overrightarrow{\mathbf{k}}_{\perp}\right\}\right) \gamma^{+}+E_{1}\left(x, \xi, t ;\left\{\overrightarrow{\mathbf{k}}_{\perp}\right\}\right) \frac{i \sigma^{+\Delta_{\perp}}}{2 m_{N}}\right. \\
& \left.\quad+E_{2}\left(x, \xi, t ;\left\{\overrightarrow{\mathbf{k}}_{\perp}\right\}\right) \frac{i \sigma^{+k_{\perp}}}{2 m_{N}}+E_{3}\left(x, \xi, t ;\left\{\overrightarrow{\mathbf{k}}_{\perp}\right\}\right) \bar{P}^{+} \frac{i \sigma^{k_{\perp} \Delta_{\perp}}}{2 m_{N}^{3}}\right] \\
& \quad \times u\left(p_{1}\right)+\cdots,
\end{aligned}
$$

where $\left\{\overrightarrow{\mathbf{k}}_{\perp}\right\}$ shortens the scalar product set involving $\overrightarrow{\mathbf{k}}_{\perp}^{2}$ and $\overrightarrow{\mathbf{k}}_{\perp} \overrightarrow{\boldsymbol{\Delta}}_{\perp}$.

In Eq. (71), the functions $H$ and $E_{i}$ depend on the futurepointed Wilson line and we omit the other possible parametrizing functions that are irrelevant for our study.

Focusing on the function $E_{2}$, as mentioned above, the time-reversal transforms give us the following property (see Appendix B for the details),

$$
E_{2}^{[ \pm]}\left(x, \xi, t ;\left\{\tilde{\overrightarrow{\mathbf{k}}}_{\perp}\right\}\right)=E_{2}^{[\mp]}\left(x, \xi, t ;\left\{\overrightarrow{\mathbf{k}}_{\perp}\right\}\right),
$$

and, therefore, we have

$$
\begin{aligned}
& E_{2}^{(\Re)[ \pm]}\left(x, \xi, t ;\left\{\tilde{\overrightarrow{\mathbf{k}}}_{\perp}\right\}\right)=E_{2}^{(\Re)[\mp]}\left(x, \xi, t ;\left\{\overrightarrow{\mathbf{k}}_{\perp}\right\}\right), \\
& E_{2}^{(\Im)[ \pm]}\left(x, \xi, t ;\left\{\tilde{\overrightarrow{\mathbf{k}}}_{\perp}\right\}\right)=-E_{2}^{(\Im)[\mp]}\left(x, \xi, t ;\left\{\overrightarrow{\mathbf{k}}_{\perp}\right\}\right) .
\end{aligned}
$$

In the $k_{\perp}$-integrated case, the function $E_{2}$ contributes to the standard twist-2 function $E(x, \xi, t)$, parametrizing the collinear hadron matrix element, as [21]

$E(x, \xi, t) \Leftarrow \int\left(d^{2} \overrightarrow{\mathbf{k}}_{\perp}\right) \frac{\overrightarrow{\mathbf{k}}_{\perp} \overrightarrow{\boldsymbol{\Delta}}_{\perp}}{\overrightarrow{\boldsymbol{\Delta}}_{\perp}^{2}} E_{2}^{(\Re)[+]}\left(x, \xi, t ;\left\{\overrightarrow{\mathbf{k}}_{\perp}\right\}\right)$.

Hence, we can conclude that in respect to the $\overrightarrow{\mathbf{k}}_{\perp} \overrightarrow{\boldsymbol{\Delta}}_{\perp}$ dependence, the real part of function $E_{2}$ is transforming as the odd function, while the imaginary part of function $E_{2}$ is transforming as the even function, i.e.,

$$
\begin{aligned}
& E_{2}^{(\Re)[ \pm]}\left(x, \xi, t ;\left\{\overrightarrow{\mathbf{k}}_{\perp}\right\}\right) \sim \phi\left(\left(\overrightarrow{\mathbf{k}}_{\perp} \overrightarrow{\boldsymbol{\Delta}}_{\perp}\right)^{2 n+1}\right), \\
& E_{2}^{(\Im)[ \pm]}\left(x, \xi, t ;\left\{\overrightarrow{\mathbf{k}}_{\perp}\right\}\right) \sim \varphi\left(\left(\overrightarrow{\mathbf{k}}_{\perp} \overrightarrow{\boldsymbol{\Delta}}_{\perp}\right)^{2 n}\right) .
\end{aligned}
$$

On the other hand, in the forward limit defined by $\Delta=0$, the imaginary part of $E_{2}$ can be related to the Sivers function $f_{1 T}^{\perp[ \pm]}[21]$ as

$$
\begin{aligned}
& \left.E_{2}^{(\Im)[ \pm]}\left(x, \xi ; \overrightarrow{\mathbf{k}}_{\perp}^{2}, \overrightarrow{\mathbf{k}}_{\perp} \overrightarrow{\boldsymbol{\Delta}}_{\perp}\right)\right|_{\Delta=0} \\
& \quad=E_{2}^{(\Im)[ \pm]}\left(x, 0 ; \overrightarrow{\mathbf{k}}_{\perp}^{2}, 0\right)=f_{1 T}^{\perp[ \pm]}\left(x ; \overrightarrow{\mathbf{k}}_{\perp}^{2}\right) .
\end{aligned}
$$

We now express the GTMD as the direct Radon transform of the $k_{\perp}$-dependent DD function $e_{2}\left(\alpha, \beta ;\left\{\overrightarrow{\mathbf{k}}_{\perp}\right\}\right)$; we have

$$
\begin{aligned}
E_{2}\left(x, \xi, t ;\left\{\overrightarrow{\mathbf{k}}_{\perp}\right\}\right) & =\mathcal{R}_{x, \xi}\left[e_{2}\left(\alpha, \beta ;\left\{\overrightarrow{\mathbf{k}}_{\perp}\right\}\right)\right], \\
e_{2}\left(\alpha, \beta ;\left\{\overrightarrow{\mathbf{k}}_{\perp}\right\}\right) & =e_{2}^{(\Re)}\left(\alpha, \beta ;\left\{\overrightarrow{\mathbf{k}}_{\perp}\right\}\right)+i e_{2}^{(\Im)}\left(\alpha, \beta ;\left\{\overrightarrow{\mathbf{k}}_{\perp}\right\}\right) .
\end{aligned}
$$

The $k_{\perp}$-dependent DD functions can be parametrized in a similar manner as has been done for the usual DD functions (cf., [7]). For instance, focusing on the imaginary part of $e_{2}\left(\alpha, \beta ;\left\{\overrightarrow{\mathbf{k}}_{\perp}\right\}\right)$, we introduce the following representation:

$$
\begin{aligned}
& e_{2}^{(\Im)[\mathcal{C}]}\left(\alpha, \beta ;\left\{\overrightarrow{\mathbf{k}}_{\perp}\right\}\right) \\
& \quad=f_{1 T}^{\perp[\mathcal{C}]}\left(\alpha ; \overrightarrow{\mathbf{k}}_{\perp}^{2}\right) \varphi\left(\left(\overrightarrow{\mathbf{k}}_{\perp} \overrightarrow{\boldsymbol{\Delta}}_{\perp}\right)^{2 n}\right) \pi(\alpha, \beta),
\end{aligned}
$$

with the normalization condition

$$
\int_{-1+|\alpha|}^{1-|\alpha|} d \beta \pi(\alpha, \beta)=1
$$

The function $\pi(\alpha, \beta)$ describes how the longitudinal momentum transfer is shared between the partons. The shape of the $\pi$-function usually is similar to shape of a hadron distribution amplitude. In the parametrization of $e_{2}^{(\Im)}$, see Eq. (80), due to the normalization conditions given by Eqs. (81) and (78) [the latter leads to $\varphi(0)=1$ ], $f_{1 T}^{\perp[\mathcal{C}]}\left(\alpha ; \overrightarrow{\mathbf{k}}_{\perp}^{2}\right)$ is treated as the well-known Sivers function associated with the Wilson line $\mathcal{C}$.

Concerning the inverse Radon transforms, for further convenience, we introduce the shortened notation for the corresponding integration measures $\left[d \mu_{A}\right.$ is the integration measure associated with the additional new term, and $d \mu_{S}$ implies the integration measure related to the standard term; see e.g., Eq. (16)] as 


$$
\begin{aligned}
& \int d \mu_{A}\{\ldots\}=\pi \int_{-\infty}^{+\infty}(d z) \delta(z) \int_{-\infty}^{+\infty} d \xi \frac{\partial}{\partial z}[\ldots] \\
& \int d \mu_{S}\{\ldots\}=\int_{-\infty}^{+\infty}(d z) \frac{\mathcal{P}}{z^{2}} \int_{-\infty}^{+\infty} d \xi[\ldots]
\end{aligned}
$$

With these notations, we have

$$
\begin{aligned}
f_{1 T}^{\perp[ \pm]} & \left(\alpha ; \overrightarrow{\mathbf{k}}_{\perp}^{2}\right) \varphi\left(\left(\overrightarrow{\mathbf{k}}_{\perp} \overrightarrow{\boldsymbol{\Delta}}_{\perp}\right)^{2 n}\right) \pi(\alpha, \beta) \\
= & -\int d \mu_{A}\left\{E_{2}^{(\Re)[ \pm]}\left(z+\alpha+\xi \beta, \xi ;\left\{\overrightarrow{\mathbf{k}}_{\perp}\right\}\right)\right\} \\
& -\int d \mu_{S}\left\{E_{2}^{(\Im)[ \pm]}\left(z+\alpha+\xi \beta, \xi ;\left\{\overrightarrow{\mathbf{k}}_{\perp}\right\}\right)\right\} .
\end{aligned}
$$

From these, we can see that the inverse Radon transformations mix up the real and imaginary parts of GTMDs thanks to the presence of the additional term that includes the integration over the measure $d \mu_{A}$. This is our principal result of the present study. In the phenomenological application, the GTMDs can be computed within a certain model. Hence, thanks to our main result, we can restore the Sivers function in an alternative way, involving the newfound additional contribution, to be compared with the available experimental data [22].

\section{CONCLUSIONS}

We now summarize the main results obtained in our paper as follows.

Having considered the Radon transforms with an arbitrary real origin function $f$, we have extended the standard representation of the inverse Radon transform up to the physically motivated case that leads to the certain restrictions for the angular (slope) Radon parameter. The standard representation of the inverse Radon transform is known in the mathematical literature [15] where the integration over the full angular region $(\varphi \in[0,2 \pi])$ has been performed by construction. As a result, first, both the origin function $f$ and its Radon transform have to be considered as the complex functions and, second, the angular restrictions allow the additional term in Eq. (17) to exist.

By considering the mathematical example where the origin function variables $x_{1}$ and $x_{2}$ are restricted but not related to each other by any extra condition, we have studied the influences of the restricted support of the origin function $f$ on their Fourier and Radon transformations. We have demonstrated that, in the most general case, the restricted support of $f$ leads to the restrictions in the $q$-plane where the Fourier image of $f$ has been determined. As a consequence, using the Fourier slice theorem, we have obtained in Sec. II B the restrictions on the related angular Radon parameter.

Then, we have revisited the problems related to the (inverse) Radon transforms, which connect the DDs with GPDs. Working with the support theorem for the case of the origin DD function where the variables $\alpha$ and $\beta$ are restricted and related by the condition $\alpha+\beta<1$ (this is the so-called spectral property), we have shown in Sec. III A that the angular restriction for the Radon parameter, $\varphi \in[0, \pi]$, takes place in a similar manner as for the considered mathematical example and leads to the existence of the additional term. Moreover, in the frame of the obtained angular restriction, the conditions (52) that stem from the spectral property of $\alpha$ and $\beta$ cannot ensure, generally speaking, the physical restrictions for the GPD parameters $z$ and $\xi$ unless we have imposed $\xi \leq 1$ from the very beginning. In other words, the spectral property of $\alpha$ and $\beta$, see Eq. (49), cannot result directly in the stronger restrictions for the GPD parameter $z$ and $\xi$ that lead them to their physical values.

Besides, we have investigated the symmetry properties of GPDs that are consistent with the existence of the additional term in Eq. (16). We have discussed the evidences pointing out that this additional term can be related to the $T$-odd effects.

Finally, we have given the physical interpretation of the new additional term that we have found. We have argued that the additional term in Eq. (16) is essentially related to the $k_{\perp}$-dependent parton distributions. Making the ansatz (80) for the transverse-momentum dependent DD function, we have presented the relations between the additional term and the Sivers function given by Eq. (83).

\section{ACKNOWLEDGMENTS}

We thank D. Müller, M. V. Polyakov, O. V. Teryaev, and colleagues from the Theoretical Physics Division of NCBJ (Warsaw) for useful discussions. The work by I. V. A. was supported by the Bogoliubov-Infeld Program. I. V. A. also thanks the Theoretical Physics Division of NCBJ (Warsaw) for warm hospitality. L. S. is supported by Grant No. 2017/ 26/M/ST2/01074 of the National Science Center in Poland and by funding from the European Union's Horizon 2020 research and innovation program under Grant No. 824093.

\section{APPENDIX A: REGULARIZATION FOR THE RADON INVERSION}

We dwell on the discussion of the problem, known as the ill posedness, which appears when we need the inversion of Radon transformations. To begin with, we introduce the socalled dual Radon transformation defined as

$$
\begin{aligned}
\mathcal{R}_{*}[g](\overrightarrow{\mathbf{x}}) & =\int_{-\infty}^{+\infty} d \tau \int_{0}^{2 \pi} d \varphi g(\tau, \varphi) \delta\left(\tau-\left\langle\overrightarrow{\mathbf{n}}_{\varphi}, \overrightarrow{\mathbf{x}}\right\rangle\right) \\
& =\int_{0}^{2 \pi} d \varphi g\left(\left\langle\overrightarrow{\mathbf{n}}_{\varphi}, \overrightarrow{\mathbf{x}}\right\rangle, \varphi\right) .
\end{aligned}
$$

Assuming that $g(\tau, \varphi)$ is being $\mathcal{R}[f](\tau, \varphi)$, we derive

$$
\mathcal{R}_{*} \mathcal{R}[f](\overrightarrow{\mathbf{x}})=\int_{0}^{2 \pi} d \varphi \mathcal{R}[f](\langle\overrightarrow{\mathbf{n}} \varphi, \overrightarrow{\mathbf{x}}\rangle, \varphi) \stackrel{\text { def }}{=} \mathcal{B}[f](\overrightarrow{\mathbf{x}}),
$$


where $\mathcal{B}[f](\overrightarrow{\mathbf{x}})$ denotes the back-projection for the Radon transform.

Let $\langle f, g\rangle$ be a scalar product defined on the suitable space. Therefore, we have

$$
\begin{aligned}
\left\langle f, \mathcal{R}_{*} \mathcal{R}[g]\right\rangle & =\left\langle\mathcal{F}[f], \mathcal{F}\left[\mathcal{R}_{*} \mathcal{R}[g]\right]\right\rangle \\
& =\int\left(d^{2} \overrightarrow{\mathbf{q}}\right) \overline{\mathcal{F}[f](\overrightarrow{\mathbf{q}})} \mathcal{F}\left[\mathcal{R}_{*} \mathcal{R}[g]\right](\overrightarrow{\mathbf{q}}) \\
& =\int_{0}^{2 \pi} d \varphi \int_{0}^{+\infty} d \lambda \lambda \overline{\mathcal{F}[f]\left(\lambda \overrightarrow{\mathbf{n}}_{\varphi}\right)} \mathcal{F}\left[\mathcal{R}_{*} \mathcal{R}[g]\right]\left(\lambda \overrightarrow{\mathbf{n}}_{\varphi}\right) .
\end{aligned}
$$

On the other hand, we can write this equation in the following equivalent form:

$$
\begin{aligned}
\left\langle f, \mathcal{R}_{*} \mathcal{R}[g]\right\rangle & =\langle\mathcal{R}[f], \mathcal{R}[g]\rangle \\
& =\int_{0}^{2 \pi} d \varphi \int_{-\infty}^{+\infty} d \tau \overline{\mathcal{R}[f](\tau, \varphi)} \mathcal{R}[g](\tau, \varphi) \\
& =\int_{0}^{2 \pi} d \varphi \int_{0}^{+\infty} d \lambda \overline{\mathcal{F}[f]\left(\lambda \overrightarrow{\mathbf{n}}_{\varphi}\right)} \mathcal{F}[g]\left(\lambda \overrightarrow{\mathbf{n}}_{\varphi}\right) .
\end{aligned}
$$

Having compared Eqs. (A3) and (A4), we obtain that

$$
\lambda \mathcal{F}\left[\mathcal{R}_{*} \mathcal{R}[g]\right]\left(\lambda \overrightarrow{\mathbf{n}}_{\varphi}\right)-\mathcal{F}[g]\left(\lambda \overrightarrow{\mathbf{n}}_{\varphi}\right)=0
$$

or

$$
\mathcal{F}\left[\mathcal{R}_{*} \mathcal{R}[g]\right](\overrightarrow{\mathbf{q}})=\frac{1}{|\overrightarrow{\mathbf{q}}|} \mathcal{F}[g](\overrightarrow{\mathbf{q}}) .
$$

Hence, if $\lambda \rightarrow+\infty$ then the inverse Radon transform is a singular (unbounded) one, i.e., $\mathcal{R}^{-1} \rightarrow+\infty$. As a result, the inverse Radon transformation needs to be regularized.

Notice that

$$
\mathcal{F}^{-1}\left[\frac{1}{|\overrightarrow{\mathbf{q}}|}\right](\overrightarrow{\mathbf{x}})=\int_{0}^{2 \pi} d \varphi \int_{0}^{+\infty} d \lambda e^{-i \lambda\left\langle\overrightarrow{\mathbf{n}}_{\varphi}, \overrightarrow{\mathbf{x}}\right\rangle},
$$

where we regularize the integration over $d \lambda$ as

$$
\lim _{\varepsilon \rightarrow 0} \int_{0}^{+\infty} d \lambda e^{-i \lambda\left(\left\langle\overrightarrow{\mathbf{n}}_{\varphi}, \overrightarrow{\mathbf{x}}\right\rangle-i \varepsilon\right)}=\lim _{\varepsilon \rightarrow 0} \frac{-i}{\left\langle\overrightarrow{\mathbf{n}}_{\varphi}, \overrightarrow{\mathbf{x}}\right\rangle-i \varepsilon} .
$$

Therefore, we have

$$
\mathcal{F}^{-1}\left[\frac{1}{|\overrightarrow{\mathbf{q}}|}\right]_{\mathrm{reg}}(\overrightarrow{\mathbf{x}})=\mathcal{R}_{*}\left[\frac{-i}{\tau-i \varepsilon}\right](\overrightarrow{\mathbf{x}}) .
$$

One can immediately see that Eq. (A9) leads to the necessity of regularization, which we have performed in
Eq. (15). To conclude this section, we have demonstrated that the ill posedness regarding the inverse Radon transformation can be treated with the help of suitable regularization in the integration over $\lambda$ as in Eq. (15).

\section{APPENDIX B: THE TIME-REVERSAL PROPERTY}

For pedagogical reasons, we present the details on how to extract the given property of function $E_{2}$ under the timereversal transform.

To begin with, we write the time-reversal transformation for the nonforward matrix element of $\gamma^{+}$-projection; we have

$$
\begin{aligned}
& \left\langle\tilde{p}_{2}, \tilde{s}_{2}\right| \bar{\psi}(0) \tilde{\gamma}^{+} \psi\left(\tilde{z}^{-}, \tilde{\mathbf{z}}_{\perp}\left|\tilde{p}_{1}, \tilde{s}_{1}\right\rangle\right. \\
& \quad=\left\{\left\langle p_{2}, s_{2}\right| \bar{\psi}(0) \gamma^{+} \psi\left(z^{-}, \mathbf{z}_{\perp}\left|p_{1}, s_{1}\right\rangle\right\}^{*},\right.
\end{aligned}
$$

where

$$
\tilde{\gamma}^{+}=\mathbb{} \gamma^{+} \mathbb{T}=\gamma^{-} .
$$

Let us write the lhs of Eq. (B1) with the help of parametrization through the function $E_{2}$. It reads

$$
\begin{aligned}
& \left\langle\tilde{p}_{2}, \tilde{s}_{2}\right| \bar{\psi}(0) \gamma^{-} \psi\left(\tilde{z}^{-}, \tilde{\mathbf{z}}_{\perp}\left|\tilde{p}_{1}, \tilde{s}_{1}\right\rangle\right. \\
& =\int\left(d \tilde{k}^{+} d^{2} \tilde{\overrightarrow{\mathbf{k}}}_{\perp}\right) e^{-i \tilde{K}^{+} \tilde{z}^{-}+i \tilde{\overrightarrow{\mathbf{k}}}_{\perp}} \tilde{\mathbf{z}}_{\perp} E_{2}\left(\tilde{k}^{+}, \tilde{\Delta}^{+} ;\left\{\tilde{\overrightarrow{\mathbf{k}}}_{\perp}\right\}\right) \\
& \times \frac{1}{2 m_{N}} \bar{u}\left(\tilde{p}_{2}, \tilde{s}_{2}\right) i \sigma^{-\tilde{\overrightarrow{\mathbf{k}}}_{\perp}} u\left(\tilde{p}_{1}, \tilde{s}_{1}\right) .
\end{aligned}
$$

Then, using $\mathbb{T}^{2}=1$, we get

$$
\begin{aligned}
& \bar{u}\left(\tilde{p}_{2}, \tilde{s}_{2}\right) \mathbb{\mathbb { N }}\left\{\mathbb{\llbracket} i \sigma^{-\tilde{\mathbf{k}}_{\perp}} \mathbb{T}\right\} \mathbb{} u\left(\tilde{p}_{1}, \tilde{s}_{1}\right) \\
& \quad=\left\{\bar{u}\left(p_{2}, s_{2}\right) i \sigma^{+\overrightarrow{\mathbf{k}}_{\perp}} u\left(p_{1}, s_{1}\right)\right\}^{*},
\end{aligned}
$$

which coincides with the spinor structure of the rhs of Eq. (B1). As is well known, the time-reversal transforms convert the dominant plus light-cone direction to the dominant minus light-cone direction. Because of the fact that the variables $x$ and $\xi$ are invariant on the light cone,

$$
x=\frac{k^{+}}{\bar{P}^{+}}=\frac{k_{+}}{\bar{P}_{+}}, \quad-2 \xi=\frac{\Delta^{+}}{\bar{P}^{+}}=\frac{\Delta_{+}}{\bar{P}_{+}},
$$

we obtain the time-reversal property as

$$
E_{2}^{[ \pm]}\left(x, \xi, t ;\left\{\tilde{\overrightarrow{\mathbf{k}}}_{\perp}\right\}\right)=E_{2}^{[\mp] *}\left(x, \xi, t ;\left\{\overrightarrow{\mathbf{k}}_{\perp}\right\}\right) .
$$

In the same manner we can derive the analogous properties for the other parametrizing functions. 
[1] D. Müller, D. Robaschik, B. Geyer, F.-M. Dittes, and J. Hořejši, Fortschr. Phys. 42, 101 (1994).

[2] X. D. Ji, Phys. Rev. D 55, 7114 (1997).

[3] A. V. Radyushkin, Phys. Rev. D 56, 5524 (1997).

[4] P. Ball and V. M. Braun, Phys. Rev. D 54, 2182 (1996).

[5] V. M. Braun, S. E. Derkachov, G. P. Korchemsky, and A. N. Manashov, Nucl. Phys. B553, 355 (1999).

[6] M. Diehl, Phys. Rep. 388, 41 (2003).

[7] A. V. Belitsky and A. V. Radyushkin, Phys. Rep. 418, 1 (2005).

[8] O. V. Teryaev, Phys. Lett. B 510, 125 (2001).

[9] D. Müller, arXiv:1711.09932.

[10] N. Chouika, C. Mezrag, H. Moutarde, and J. RodríguezQuintero, Eur. Phys. J. C 77, 906 (2017).

[11] N. Chouika, C. Mezrag, H. Moutarde, and J. RodríguezQuintero, EPJ Web Conf. 137, 05020 (2017).

[12] N. Chouika, Generalized parton distributions and their covariant extension: Towards nucleon tomography, Ph.D. thesis, University of Paris-Saclay, 2018.

[13] H. Moutarde, Nucleon reverse engineering: Structuring hadrons with colored degrees of freedom, Habilitation thesis, University of Paris-Saclay, 2015, p. 243.
[14] S. R. Deans, The Radon Transform and Some of Its Applications (Wiley, New York, 1983), p. 299.

[15] I. M. Gelfand, M. I. Graev, and N. Ya. Vilenkin, Generalized Functions, Volume 5: Integral Geometry and Representation Theory (AMS Chelsea Publishing: An Imprint of the American Mathematical Society, 1966), p. 449.

[16] Notice also that it is sometimes useful to present the Radon transform in the form of $\mathcal{R}[f](\tau, \varphi)=\mathcal{R}[f](z, \xi) /|\cos \varphi|$, where $z=\tau / \cos \varphi, \xi=\tan \varphi \equiv q_{2} / q_{1}$.

[17] J. Boman and E. T. Quinto, Duke Math. J. 55, 943 (1987).

[18] I. R. Gabdrakhmanov, D. Müller, and O. V. Teryaev, arXiv: 1906.01458.

[19] D. Boer, P. J. Mulders, and F. Pijlman, Nucl. Phys. B667, 201 (2003).

[20] S. Meissner, A. Metz, and M. Schlegel, arXiv:0807 .1154 .

[21] S. Meissner, A. Metz, and M. Schlegel, J. High Energy Phys. 08 (2009) 056.

[22] I. V. Anikin and L. Szymanowski (to be published). 